\title{
Der Umgang mit Transsexualität in der Europäischen Union unter besonderer Berücksichtigung von Belgien
}

Jan Steinmetzer und Dominik Groß

\section{Einleitung}

Transsexualität bzw. Transsexualismus ist laut ICD-10, der „Internationalen Klassifizierung von Krankheiten“ der Weltgesundheitsorganisation (WHO), eine Form der Geschlechtsidentitätsstörung. Sie liegt vor, wenn ein Mensch körperlich eindeutig dem männlichen oder weiblichen Geschlecht zugordnet werden kann, sich jedoch als Angehöriger des anderen Geschlechts empfindet und danach strebt, sich diesem Geschlecht auch körperlich weitestgehend anzugleichen.

Bei vielen transsexuellen bzw. transidenten ${ }^{2}$ Menschen nimmt die psychische Belastung in Pubertät und Adoleszenzphase deutlich zu. Ein Teil der Betroffenen trifft schließlich die Entscheidung, die Geschlechterrolle zu wechseln. Der Wechsel der Geschlechtszugehörigkeit führt dabei vielfach zu sozialen Problemen. Nicht selten zerbrechen Partnerschaften, es finden familiäre

1 Der vorliegende Beitrag entstand in Rahmen eines am Universitätsklinikum Aachen durchgeführten, auf zwei Jahren angelegten Forschungsprojekts mit dem Titel „Ethische Fragen im Umgang mit Transsexualität“ (STARTProjekt).

2 Ein Teil der Betroffenen favorisiert mittlerweile den Begriff „Transidentität“. Der Begriff „Transidentität“ soll im Unterschied zu Transsexualität - deutlich machen, dass eine Störung der (Geschlechts-)ldentität und kein sexuelles Problem im Sinne „sexueller Präferenzen“ oder „sexueller Orientierung“ vorliegt. Vgl. hierzu auch Steinmetzer/Groß/Duncker (2006). Andere Betroffene favorisieren die Bezeichnung „Transgender(ismus)“. Da keine Bezeichnung ungeteilte Zustimmung erfährt und da der Terminus „Transsexualität“ in Deutschland bis heute sowohl im allgemeinen Sprachgebrauch als auch mit Blick auf die einschlägige Gesetzgebung dominiert, benutzen wir unbeschadet der terminologischen Unschärfen diesen etablierten Begriff. 
Ausgrenzungen statt und/oder der Arbeitsplatz ist bedroht. ${ }^{3}$ Letztlich hängt der Umgang mit Transsexualität sehr stark vom persönlichen Umfeld und dem soziokulturellen Kontext ab. Der gesellschaftliche Umgang mit dem Phänomen wird wiederum von den jeweiligen (eher liberalen bzw. eher restriktiven) rechtlichen Rahmenbedingungen beeinflusst, aber auch von der medizinischen Deutung des Phänomens „Transsexualität“. So wirft bereits die derzeit vorherrschende Einordnung der Transsexualität als Krankheit Probleme auf: Während sie einerseits die Voraussetzung für medizinische Maßnahmen und deren Finanzierung durch die Krankenkassen darstellt, bringt sie andererseits eine Abhängigkeit der Betroffenen von ärztlichen Gutachten und richterlichen Urteilen - und damit eine Einschränkung der Selbstbestimmtheit - mit sich. Vor allem begründet die Einstufung der Transsexualität als Störung der Geschlechtsidentität (ICD-10) eine Pathologisierung des transsexuellen Empfindens, die u. U. empfindliche Rückwirkungen auf das Selbstbewusstsein und das Selbstbild der Betroffenen zeigt, aber auch Berührungsängste bei anderen Menschen begründen bzw. verstärken kann.

Was die juristischen Rahmenbedingungen betrifft, so lassen sich spätestens seit Mitte der 199oer Jahre innerhalb der Europäischen Union deutliche Liberalisierungstendenzen nachweisen, die auf Urteile des Europäischen Gerichtshofs (EuCH) zurückgehen. Dieser sieht die Wahrung der Crundrechte der Bürger der Mitgliedsstaaten der Europäischen Union als wesentliche Aufgabe an. ${ }^{4}$ Zudem forderte das Europäische Parlament die Mitgliedstaaten 1989 in einem Entschließungsantrag dazu auf, die Rechte von transsexuellen Menschen anzuerkennen. ${ }^{5}$ Verwiesen wird vielfach auch auf $\mathbb{\$} 12$ der Europäischen Menschenrechtskonvention (EMRK), ${ }^{6}$ der für jeden Menschen das Recht auf Eheschließung vorsieht.

Vor dem Hintergrund dieser Entwicklung soll im vorliegenden Beitrag der Frage nachgegangen werden, wie sich der Umgang mit Transsexualität in Belgien, das zu den Gründungsstaaten der EWG (1957) gehört und den Europarat, den Ministerrat und die Europäische Kommission beherbergt, darstellt. Dabei sollen juristische, soziale und medizinische Aspekte im Königreich berücksichtigt werden (s. Kap. 2). Anschließend erfolgt ein Vergleich des juristischen und sozialen Umgangs mit dem Phänomen Transsexualität mit anderen europäischen Ländern der EU, namentlich Frankreich, England und Portugal (s. Kap. 3).

3 Pfäfflin (2006).

4 Das Urteil EuGH Rs C-13/94 „P. gegen S. und Cornwall County Council“ "gilt als weltweit erster Fall, in dem ein Schutz vor Diskriminierung bei Vorliegen von Transsexualität angenommen wurde." Greif (2005), S. 139. - Vgl. auch Whittle, www.pfc.org.uk/node/364 (2004).

5 ABl 1989 C 256/33. Verweise darauf finden sich z. B. in: BVerfG, 1 BvL 1/04 vom 18.7.2006, Absatz-Nr. 54 bzw. www. dgti.org/recht/euro2.htm.

6 http://conventions.coe.int/Treaty/ger/Treaties/Html/005.htm. 


\section{Der Umgang mit Transsexualität in Belgien}

\subsection{Juristische und gesellschaftliche Aspekte}

In Belgien bestanden - trotz gegenteiliger Aussagen ${ }^{7}$ - bis in die jüngste Vergangenheit hinein keine gesetzlichen Regelungen zum Umgang mit Transsexualität bzw. mit der Geschlechtsumwandlung. Entsprechende rechtliche Fragen (z. B. die Änderung des Geburtsregisters betreffend) wurden vielmehr bis ins Jahr 2007 durch gerichtliche Urteile entschieden. ${ }^{8}$

Erst vor kurzem wurde nach langjährigen Beratungen eine gesetzliche Regelung zur Änderung des Geschlechts sowie des Vornamens eingebracht und verabschiedet: Das „Loi relative à la Transsexualité“" wurde nach dem Vorbild des liberalen britischen „Gender Bill Recognition Act “"10 konzipiert. Nachdem der Senat dem ursprünglichen Entwurf Änderungen hinzugefügt hatte, genehmigte die parlamentarische Kommission die revidierte Fassung am 11. April 2007. Die abschließende Abstimmung über das „Loi relative à la Transsexualité“ im belgischen Parlament vor der Unterzeichnung durch den König fand am 26. April 2007 statt. Für den Gesetzentwurf stimmten 93 Abgeordnete, 15 votierten dagegen und 28 enthielten sich der Stimme. Nach der - bis zur Drucklegung dieses Aufsatzes noch nicht vorgenommenen - Unterzeichnung durch den König wird das Gesetz im Staatsblatt veröffentlicht werden und nach einem Ablauf von 6o Tagen in Kraft treten. Es soll Erleichterungen bei der Änderung des Namens und Geschlechtseintrags bieten:

„La proposition de loi de Mme Vautmans et consorts [...] introduit essentiellement une simplification pour les transsexuels des procédures administratives visant à modifier leur prénom, dès le début du traitement hormonal, et à changer la mention de leur sexe, après intervention chirurgicale, sur leur acte de naissance et leur carte d'identité afin de rendre ces documents conformes à leur nouvelle réalité anatomique. ${ }^{\text {“11 }}$

Darüber hinaus wird im neuen Gesetz eine enge Zusammenarbeit zwischen den Fachärzten der Disziplinen Chirurgie, Psychiatrie und Endokrinologie festgeschrieben.

Doch welche Entwicklung ging dieser aktuellen gesetzlichen Neuregelung voraus? Der juristische Umgang mit Transsexualität in Belgien ist seit Jahrzehnten eng verknüpft mit richtungsweisenden Entscheidungen auf europäischer Ebene. Einen Meilenstein in der Rechtsprechung in Belgien und Europa markierte der Fall Van Oosterwijck aus dem Jahr 1979. Es handelte sich um einen der ersten Fälle europaweit, der die juristische Anerkennung (in diesem

7 „In Belgien besteht eine Gesetzgebung zur Transsexualität, die permanent angepasst wird." Zitiert nach www.4ftm.de/ppt/rechtliche_situation_ts_europa.ppt.

8 Auch in anderer Hinsicht bietet Belgien besondere Rahmenbedingungen: In Belgien ist die Partnerschaft zwischen gleichgeschlechtlichen Partnern der Ehe zwischen Angehörigen unterschiedlichen Geschlechts rechtlich gleichgestellt: Siehe dazu Pintens/Scherpe (2003) sowie Pintens/Scherpe (2004).

9 www.dekamer.be/kvvcr/showpage.cfm?section=flwb\&language=nl\&cfm=/site $/$ wwwcfm/flwb/flwbn. $\mathrm{cfm}$ ?dossierlD=0903\&legislat=51\&inst=K

10 Zur Situation in Großbritannien vgl. auch das folgende Kapitel.

11 www.dekamer.be/FLWB/PDF/51/0903/51K0903002.pdf. 
Fall bereits durchgeführter) geschlechtsangleichender Maßnahmen einforderte. Ihn nahm der Europäische Gerichtshof für Menschenrechte (EGMR) zum Anlass, um zum ersten Mal das individuelle bürgerliche Grundrecht auf die persönliche Wahl der Geschlechtsidentität festzustellen. ${ }^{12}$ Seit dem Jahr $1985^{13}$ werden Klagen auf Änderung der Geschlechtszugehörigkeit in Belgien im Allgemeinen positiv beschieden. Einzelne Urteile fielen dennoch auch in der Folgezeit ablehnend aus. ${ }^{14}$ Das Europäische Parlament - in Brüssel und Straßburg situiert - gab zudem im Jahr 1989 eine „Erklärung zur Diskriminierung von Transsexualität" ab, und der Europäische Gerichtshof interpretierte 1996 die Weigerung, eine Geschlechtsänderung juristisch anzuerkennen, als Diskriminierung, die auf dem Geschlecht basiere und damit einen Verstoß gegen die Grundrechte, die in den Verfassungen der Staaten der Europäischen Union niedergelegt und garantiert sind, darstelle. ${ }^{15}$ Darüber hinaus vertrat der Europäische Gerichtshof für Menschenrechte nach mehreren vorherigen Urteilen letztmals 2003 die Auffassung, dass die Weigerung, die Geschlechtsänderung einer transsexuellen Person rechtlich anzuerkennen, als Verstoß gegen das Recht auf Selbstbestimmtheit des privaten Lebens anzusehen sei.

Angesichts der beschriebenen Tendenzen in der europäischen Rechtsprechung gab es seit dem Jahr 2004 in Belgien Bemühungen mit dem Ziel, rechtliche Rahmenbedingungen für die Anerkennung von Transsexualität auf gesetzlichem Weg zu schaffen. Erklärtes Ziel des Gesetzentwurfs, der nach der Abgeordneten Hilde Vautmans benannt wurde, ist der leichtere Weg zu gesetzlicher Anerkennung für Betroffene, wobei gleichzeitig Missbrauch verhindert werden soll:

„On nous répond que le Législateur veut faciliter les démarches liées au changement d'état civil pour les personnes transsexuelles mais aussi éviter des abus, car le transsexualisme a des implications au niveau de l'état civil, une matière qui est régie par l'Etat. ${ }^{[17}$

Allerdings wurde der Entwurf von Seiten der Betroffenen kritisch begleitet. ${ }^{18}$

Da die neue Gesetzgebung ihre Bewährungsprobe noch vor sich hat, möchten wir im Folgenden eine Übersicht über den bisherigen Umgang mit Transsexualität in Belgien geben:

Wenn eine Störung der Geschlechtsidentität diagnostiziert wird, schließt sich im Regelfall eine psychologische, hormonelle und chirurgische Behandlung an. In Belgien (wie übrigens auch in den Niederlanden) trägt die staatliche Krankenversicherung alle Kosten, die durch die Begutachtung und Be-

12 Greif (2005), S. $63 \mathrm{f}$.

13 Urteil Gericht Brüssel vom 23.1.1985, in: Revue trimestrielle du droit familial 1987, S. 283.

14 Pintens (2004), S. 23.

15 Greif (2005), S. 130.

16 Greif (2005), S. $115 \mathrm{ff}$.

17 Synthèse de l'entrevue du 22 avril 2005 entre députés et associations sur la proposition de loi sur la transsexualité, www.trans-action.org/pdf/compte_rendu_Parl_synthese_22042005.pdf [21.05.2007]

18 www.petitiononline.com/betslaw [21.05.2007]. In diesem Zusammenhang betonten Betroffene, dass sie einer am Einzelfall orientierten Rechtsprechung den Vorzug gegenüber einer übereilten und unangebrachten Gesetzgebung geben würden: „De plus, les personnes transsexuelles disposent actuellement d'une bonne jurisprudence qui est préférable à une mauvaise loi." - www.trans-action.org/pdf/position-paper2.pdf [21.05.2007]. 
handlung von Transsexualität entstehen. Dies gilt auch für Kinder und Jugendliche unter 18 Jahren. Allerdings ist für Menschen unter 18 Jahren - dies weicht von der Situation in den Niederlanden ab - nur eine psychologische Behandlung vorgesehen.

Vorbedingung für einen juristischen Wechsel der Geschlechtszugehörigkeit ist und bleibt die genitalchirurgische Anpassung. ${ }^{19}$ Die Verfahrensdauer bis zur dann möglichen Änderung der Geburtsurkunde beträgt mehr als ein Jahr und wurde bisher durch Gerichte verfügt. Nach Änderung der Geburtsurkunde ist es möglich, andere offizielle Dokumente (Personalausweis, Führerschein, Reisepass und Sozialversicherungsausweis) ebenfalls umschreiben zu lassen.

Transsexuelle Menschen in Belgien sehen sich in verschiedener Hinsicht benachteiligt: So verlieren vor der Geschlechtsumwandlung bestehende Ehen (wie in den meisten europäischen Staaten) ihre Gültigkeit. Einer Betroffenen, die zuvor ein Mann war („Mann-zu-Frau-Transsexuelle“ oder kurz „Transfrau“), steht lediglich die Möglichkeit offen, einen Mann zu heiraten bzw. umgekehrt. Allerdings ist es möglich, gleichgeschlechtliche Partnerschaften einzutragen, die ebenfalls gesetzlich anerkannt und gleichgestellt sind, aber in sozialversicherungsrechtlicher Hinsicht Probleme verursachen. Adoptionen von Paaren, bei denen ein Partner transsexuell ist, sind erlaubt. Schwierigkeiten können bei der tariflichen Einstufung bei Kranken- bzw. Lebensversicherungen entstehen, während sich die Zuteilung von Renten und Pensionen bis dato als unproblematisch erwiesen hat. Transfrauen werden in Belgien von weiblichen, Transmänner von männlichen Polizeibeamten durchsucht. Die betreffende Regelung wurde allerdings erst nach langen Auseinandersetzungen gerichtlich erzwungen. ${ }^{20}$

In Belgien existieren gegenwärtig nur wenige Schwerpunktzentren für Transsexualität, die eine Zusammenarbeit zwischen chirurgischen, psychiatrischen und endokrinologischen Fachärzten bieten. Die Kooperation in Zentren kann derzeit nur in Gent und Antwerpen geleistet werden; auf das Krankheitsbild Transsexualität spezialisierte chirurgische Fachärzte sind im frankophonen Landesteil nicht tätig. Einer dritten Gruppe in Lüttich wird angesichts einer fehlenden operativen Ausrichtung die Möglichkeit verwehrt, Behandlungen mit dem Ziel geschlechtsanpassender Maßnahmen auch nur zu beginnen. Diese Verfahrensweise bietet Anlass für Kritik, da betroffene Wallonen somit nicht über ein Behandlungszentrum verfügen, in dem sie sich mit den behandelnden Ärzten in ihrer Muttersprache verständigen können - ein Aspekt, der von wallonischen Transsexuellen als Benachteiligung (,source d'incompréhension et donc d'erreurs de jugement“) gewertet wird. ${ }^{21}$ Doch die Betroffenen bemängeln nicht nur die sprachlichen Hindernisse, sondern auch die faktische Einschränkung der freien Arztwahl durch die Zusammenstellung fester Ärzteteams. Zudem wurde die bisherige Rechtspraxis

19 Vgl. Art. 12 des neuen Gesetzes.

20 www.just.fgov.be/img_justice/publications/pdf/55.pdf [21.05.2007].

21 Synthèse de l'entrevue du 22 avril 2005 entre députés et associations sur la proposition de loi sur la transsexualité, www.trans-action.org/pdf/compte_rendu_Parl_synthese_22042005.pdf [21.05.2007]. 
bis zur aktuellen Verabschiedung des „Loi relative à la Transsexualité“ als zu restriktiv und paternalistisch empfunden:

„Les transsexuels belges craignent une atteinte aux libertés fondamentales. Ils veulent profiter de ce débat pour lever les tabous, évoquer les tentatives de suicide, les souffrances encourues, les discriminations sociales et pousser les élus à regarder vers d'autres pays, comme les Pays-Bas, plus tolérants en la matière. ${ }^{\text {(22 }}$

Auch die rechtliche Fixierung auf das vollendete 18. Lebensjahr als den frühest möglichen Behandlungsbeginn sowie die dominierende Rolle der Medizin insbesondere der Psychiatrie - im Begutachtungsprozess stießen wiederholt auf Widerspruch.

\subsection{Medizinische Aspekte}

Die Zahl der Personen, die sich bis 2005 in Belgien einer geschlechtsanpassenden Operation unterzogen, beläuft sich auf ca. 1.ooo: „Un millier d'entre eux, tout au plus, sont passés entre les mains d'un chirurgien en Belgique. ${ }^{“ 23}$ De Cuypere et al. ermittelten 1995 für Belgien dabei ein Verhältnis von 1,7 Transfrauen zu einem Transmann. ${ }^{24}$ Auch in Belgien finden sich Unstimmigkeiten hinsichtlich der Bezeichnung des Phänomens:

„Tout d'abord la définition même du transsexualisme nous semble poser problème (article 2 [de la proposition de la loi sur le transsexualisme, die Autoren]). En effet, celle-ci est rigide, normative et restrictive. D’autant qu'une telle clause revient à définir une condition médicale, ce qui devrait relever de la compétence du corps médical. “' ${ }^{\prime 2}$

Im Vorfeld des Gesetzentwurfs konnten - laut Aussage der Betroffenen - nur für den flämischen Landesteil repräsentative Aussagen über das medizinische Behandlungsprogramm getroffen werden:

„Or, les personnes et associations consultées jusqu'à notre participation n'étaient représentatives que du nord du pays. Dès lors il nous a semblé indispensable d’apporter un éclairage sur la situation dans le sud du pays. ${ }^{26}$

Während das neue Gesetz die Behandlung gemäß Artikel 3 ausschließlich in die Hände von Expertenteams gelegt wissen will, bestand in Belgien bisher hierzu keine grundsätzliche Verpflichtung. Es gibt allerdings nach wie vor nur eine geringe Anzahl von Chirurgen, die geschlechtsangleichende Operationen vornehmen. Phalloplastische Operationen werden beispielsweise der-

22 „Les transsexuels sans droits ni loi“, Le Soir (26.09.2005), p. 1, www.lesoir.be/services/archives/t-20050926-001DUR. html?firstHit=90\&cat=07007000 [21.05.2007].

23 Ebd.

24 De Cuypere/Jannes/Rubens (1995).

25 Synthèse de l'entrevue du 22 avril 2005 entre députés et associations sur la proposition de loi sur la transsexualité, www.trans-action.org/pdf/compte_rendu_Parl_synthese_22042005.pdf [21.05.2007]

26 Ebd. 
zeit $^{27}$ nur von einem Chirurgen in Gent durchgeführt. ${ }^{28}$ Darüber hinaus wird in Verlautbarungen belgischer Betroffener vielfach auf den unterschiedlichen medizinischen Umgang mit Transsexualität in den verschiedenen Landesteilen verwiesen:

„Nous avons souligné le fait que l’approche clinique (et donc le vécu des personnes transsexuelles) est très différente entre la partie francophone et la partie neerlandophone du pays. ${ }^{\text {"2 }}$

Aus diesem Grund sind z. B. belgische Transmänner fast zwingend darauf angewiesen, sich im UZ Gent ${ }^{30}$ operieren zu lassen. Aus Gründen der besseren Kenntnis der Abläufe werden dort vielfach auch die Diagnose und hormonelle Behandlung vorgenommen. Bei einer psychiatrischen Behandlung bzw. Diagnosestellung andernorts muss am Operationszentrum nur noch ein einleitendes Betreuungsgespräch geführt werden. Im Übrigen gelten auch in Belgien die Standards der HBIGDA: $:^{31}$ „La thérapie médicale en Belgique est basé sur ce SOC." 32

Im Gegensatz zur medizinischen Behandlung von Transsexualität in Deutschland, die nach einem fünfstufigen Plan abläuft, ${ }^{33}$ wird die Therapie in Belgien in nur vier Phasen durchlaufen, die sich folgendermaßen charakterisieren lassen:

Diagnostik: Im Rahmen der psychiatrischen Diagnostik wird zunächst versucht, die Intensität und Nachhaltigkeit des Wunsches nach einer Geschlechtsumwandlung zu bestimmen und ggf. den Wunsch des Patienten/der Patientin nach sofortiger hormoneller und chirurgischer Therapie abzuschwächen. Die Diagnostik wird therapeutisch in einem offenen Dialog geführt und oft von psychologischen Tests flankiert. Darüber hinaus müssen mögliche hormonale oder genetische Anomalien ausgeschlossen werden. Diese Phase der Diagnostik wird mit mindestens sechs Monaten veranschlagt.

Real-Life-Test mit hormoneller Behandlung: In diesem Stadium unterzieht sich der Patient einer Hormontherapie, bleibt aber in engem Austausch mit dem behandelnden Psychiater. Der Real-Life-Test hat, wie in anderen Ländern auch, zum Ziel, dass der Patient die angestrebte Geschlechtsrolle ganztägig lebt. Auch in Belgien gilt dies als zentraler Bestandteil der Behandlung. Der All-

\footnotetext{
27 Stand $4 / 2007$.

28 „Prof. Monstrey is eigenlijk de enige chirurg die de falloplastiek en de metaidoioplastiek uitvoert." www.transman. $\mathrm{nl} /$ genderteam.php.

$29 \mathrm{Vgl}$. Fußnote 22.

30 Universitair Ziekenhuis van Gent, Centrum voor Seksuologie en Genderproblematiek, www.uzgent.be/diensten/ dienst_93.cfm: „Het team is multidisciplinair samengesteld met twee psychiaters, twee internisten-endocrinologen, een plastisch chirurg, een uroloog, een neuro-linguist, een dermatoloog, een hoofd- en halschirurg, een vrouwenarts en verscheidene verpleegkundigen alsook een jurist."

31 Die ehemalige Harry Benjamin International Gender Dysphoria Association hat sich umbenannt und firmiert nun als The World Professional Association for Transgender Health, www.wpath.org [21.05.2007].

32 www.genderstichting.be/fr/therapie_medical.html bzw. www.genderstichting.be/StandardsOfCare_NED.pdf. Die Standards werden ausführlich beschrieben unter www.wpath.org/publications_standards.cfm.

33 Becker et al. (1998).
} 
tagstest ist auf 1,5 bis zwei Jahre festgesetzt, erst danach darf die chirurgische Behandlung erfolgen.

Nach der Bestätigung der Diagnose kann - hier zeigt sich ein wesentlicher Unterschied zu Behandlungsrichtlinien in anderen Ländern - zeitgleich mit dem Alltagstest die hormonelle Behandlung begonnen werden. Der Alltagstest wird dementsprechend in enger Verbindung mit der radikalen Änderung der Körperfunktionen absolviert. Die wichtigsten hormonell bedingten Funktionsänderungen sind ein Rückgang der Libido sowie die vielfach von Transfrauen erstrebte Haar- und Bartreduktion. Zu beobachten sind weiterhin $z$. B. eine Änderung der Körperfettstrukturen, eine weichere Haut und/oder eine Feminisierung des Gesichts. Bei Transmännern werden insbesondere eine Vermännlichung der Stimme, eine Vermehrung der Muskulatur, eine Libidoerhöhung, fettigere Haut sowie ein Wachstum der Klitoris bewirkt. Ungewollte Wirkungen können eine Zunahme der Aggressivität, Haarausfall oder Muskelschmerzen sein. Gleichwohl sind die Auswirkungen der Therapie in diesem Stadium noch umkehrbar. ${ }^{34}$

Nach dem derzeitigen medizinischen Wissensstand ist für die Betroffenen eine lebenslängliche hormonelle Behandlung notwendig. Wenn der Übergang zum anderen Geschlecht allerdings zu schnell vollzogen wird, kann dies zu schwerwiegenden psychischen Problemen führen. Aus diesem Grund sind für den Alltagstest mindestens 1,5 Jahre angesetzt.

Genitalchirurgische Operationen: Wenn die Hormontherapie zufrieden stellend verlaufen ist und die Änderung der sozialen Rolle positive Perspektiven eröffnet, kann der Übergang zu den plastischen, insbes. zu den genitalchirurgischen Operationen erfolgen. Diese beginnen frühestens zwei Jahre nach der Diagnose. Die MzF-Operation besteht aus nur einem Eingriff: Penis und Skrotum werden ausgehöhlt, während mit der Haut eine Neo-Vagina, Schamlippen und Klitoris geformt werden. Wesentlich schwieriger und langwieriger sind die FzM-Operationen: Zunächst wird eine Mastektomie durchgeführt, dann werden Gebärmutter und Ovarien entfernt. Der anschließende Aufbau einer Phalloplastik aus Muskelmaterial der Arme ist optional; trotz großer Operationsfortschritte auf diesem Gebiet bleiben die FzM-Operationen risikoreicher und bringen ästhetisch bzw. funktionell nur bedingt zufrieden stellende Resultate. Anzumerken bleibt, dass die Ermittlung des Karyotyps für die Anerkennung der geschlechtsanpassenden Operation in Belgien obligatorisch ist.

Nachsorge: Diese gliedert sich verschiedene Abschnitte. Für die entsprechende Behandlung kommt die Krankenversicherung zum Teil auf. Die Behandlung ist in die standardisierte Geschlechtsanpassung eingegliedert.

- Epilation: Die hormonelle Therapie von Transfrauen weist zwei limitierende Faktoren auf, welche die Stimme und die Gesichtsbehaarung betreffen. Die Gesichtsbehaarung kann oft erst durch eine lange und mühsame Epilation durch einen Hautarzt oder in einem Schönheitsinstitut komplett entfernt werden. Grundsätzlich wird von Betroffenenseite die

34 www.genderstichting.be. 
Laserepilation empfohlen: Sie funktioniert auf der Basis von konzentriertem Licht. Die Energie zerstört durch Hitze die Haarwurzeln nachhaltig. Je dunkler die Haare sind, desto wirksamer ist die Therapie.

- Stimmbehandlung: Wie oben angedeutet, hat die hormonelle Therapie nahezu keine Auswirkung auf die Stimme von Transfrauen. Angesichts der Bedeutung der Stimme für die weibliche Persönlichkeit gilt dies als schweres Manko. Als Ausgleich wird in Belgien ein logopädisches Training angeraten, das viel Zeit (u. U. mehrere Jahre) und Übung in Anspruch nimmt.

- Ästhetik: Auch die äußere Ästhetik betreffende operative Maßnahmen zählen zur Nachsorgebehandlung. Vor allem Änderungen am oder um das Gesicht werden ermöglicht. Neben der Entfernung von Alterserscheinungen werden Interventionen empfohlen, die den Gesichtsschnitt und seine Umrisse modifizieren. Dies können z. B. Veränderungen an Nase, Kinn, Kiefer oder an der Frontpartie sein. Dabei wird die Überzeugungskraft des männlichen bzw. weiblichen Gegenübers überwiegend vom Gesichtseindruck bzw. -schnitt bestimmt. ${ }^{35}$ Form und Umriss des Schädels beeinflussen die Positionierung der Haut maßgeblich. Vor allem das Kinn ist ein Bereich, durch den jemand als Mann oder Frau identifiziert wird. Allerdings wird von ärztlicher Seite in diesem Zusammenhang die Selbsteinschätzung des Patienten als wichtig angesehen, um einen endgültigen Behandlungsplan skizzieren zu können.

\section{Der Vergleich mit anderen europäischen ländern}

\subsection{Portugal}

In Portugal, das 1986 der Europäischen Gemeinschaft beigetreten ist, existieren bis dato keine gesetzlichen Bestimmungen zur Transsexualität. Die wenigen Gerichtsentscheide, die zum Teil bis in die 1980er Jahre zurückreichen, ergeben ein inhomogenes Bild. Eine Personenstandsänderung ist zwar aufgrund der Urteile des EGMR möglich; die soziale Lage der Betroffenen, die mitunter Angriffe gegen Leib und Leben mit einschließen kann, schränkt allerdings die Möglichkeit eines öffentlichen Lebens als transsexuelle Person erheblich ein. Die Forderung nach Beseitigung der Rechtsunsicherheit im Umgang mit Transsexualität ist bisher ${ }^{36}$ nicht erfüllt. So heißt es in einer Stellungnahme von Betroffenen:

„A situação das pessoas transexuais em Portugal, decorrente do actual quadro legislativo e clínico, em termos absolutos e em termos comparativos com a das pessoas transexuais noutros países da União Europeia, não é satisfatória. Dividindo esta situação no plano legal, clínico e social, encontramos diversos problemas, muitos dos quais partilhados pelas pessoas Transexuais noutros pontos do mundo. ${ }^{\text {“37 }}$

35 www.genderstichting.be/nl/esthetische_operaties.html.

36 Stand April 2007.

37 „Die Situation der transsexuellen Personen in Portugal in Bezug auf den aktuellen gesetzlichen und klinischen 
In einer aktuellen Überblicksdarstellung der Medizinischen Fakultät der Universität Lissabon werden Geschlechtsidentitätsstörungen nach wie vor in einen direkten Zusammenhang mit Homosexualität gebracht, ${ }^{38}$ was aus wissenschaftlicher Sicht erklärungsbedürftig erscheint. Zwar werden hier genitalanpassende Operationen durchgeführt, die den internationalen Standards entsprechen ${ }^{39}$ Im Vergleich mit anderen europäischen Ländern spielt die medizinische Behandlung von Transsexualität jedoch eine untergeordnete Rolle; dies ist auch der Tatsache geschuldet, dass ca. 70 \% der Betroffenen keinen Krankenversicherungsschutz genießen.

Aussagen von transsexuellen Personen aus Portugal lassen überdies darauf schließen, dass die Betroffenen bis zum heutigen Tag gesellschaftlich stigmatisiert werden. In einer Selbstdarstellung einer Gruppe von 50 Mitgliedern der „Transgender Community“40 bezeichnen sich $56 \%$ als „Sex-Worker“. ${ }^{41}$ Die geringe gesellschaftliche Akzeptanz transsexueller Personen spiegelt sich im Übrigen in den (in ihrer Zielsetzung wenig weit reichenden) interessenpolitischen Aktivitäten der betroffenen Personen wider. Letztere geben als vordringliches Ziel ihrer Öffentlichkeitsarbeit die „Demystifizierung“ transsexueller Personen an. ${ }^{42}$ Des weiteren scheint die HIV-Prophylaxe, dies ergibt sich aus dem Hinweis auf die hohe Zahl der Erkrankten, eines der drängendsten präventiven und damit auch medizinischen Anliegen zu sein. Daneben wurde 2001 aus Betroffenensicht die Hoffnung geäußert, dass verbindliche juristische Regularien für den gesamten Weg von der Geschlechtsanpassung bis hin zu einer Anerkennung des ,neuen' Personenstandes erlassen würden. ${ }^{43}$ Diese Hoffnung blieb allerdings bisher unerfüllt. Der Blick auf die bestehende soziale Wirklichkeit in Portugal zeigt ein Bild, das durch eine erhebliche Rechtsunsicherheit, eine begrenzte Zugänglichkeit zu medizinischen Maßnahmen und nicht zuletzt durch gesellschaftliche Stigmatisierung geprägt ist. Für transsexuelle Menschen in Portugal stellen die zweifelsfreie juristische

Zustand ist weder in absoluten Begriffen noch im Vergleich mit anderen Betroffenen in anderen Ländern der Europäischen Union zufrieden stellend. Unterteilt in die gesetzliche, klinische und soziale Situation sind verschiedene Probleme zu konstatieren, die von zahlreichen transsexuellen Personen in anderen Ländern geteilt werden.“, Associação ILGA Portugal, www.ilga-portugal.pt/glbt/gip/transexualidade.htm\#SITUA [21.05.2007] (übersetzt von den Verf.).

38 Faculdade de Medicina de Lisboa (Hrsg.) (2004) mit den Aufsätzen: Pechorro Pedro/Vieira, Rui M. X.: Avaliação Psicológica de um Grupo de Transexuais com Indicação para Cirurgia de Reatribuição de Sexo. Estudo preliminar, S. 77-84; Vieira, Rui M. X.: Transexualismo: da Clínica ao Diagnóstico, S. 85-90, Matos, Manuel Godinho de: A Cirurgia da Inter e da Transexualidade, S. 91-98.

39 Matos (2004).

40 Association For The Study And Defence Of The Right To The Genre Identity: The Portugese Transgender Community: An Unknown Reality, http://a-trans.planetaclix.pt/english_documentation/documentation.htm.

41 Z. B. http://intimidadescd.blogspot.com/2005/07/transexualidade-em-portugal.html.

42 Association For The Study And Defence Of The Right To The Genre Identity: The image of transsexuality and its connection to the HIV virus, http://tgeu.net/Documents/p_ATrans_Bangkok.pdf.

43 Coelho de Lima (2001), S. 156: „Continuamos a defender [...] que seja instituído um sistema prévio de controlo judicial para as mudanças de sexo, que deverá ser obrigatória a mudança da menção registal [...]. " „Wir werden fortfahren, uns dafür einzusetzen, dass ein System der juristischen Kontrolle für Geschlechtsänderungen eingerichtet wird, das von der Geschlechtsänderung bis zur Personenstandsänderung obligatorisch durchlaufen werden muss [...]“ (übersetzt von den Verf.). 
Anerkennung des Lebens im Wunschgeschlecht, die Verfügbarkeit medizinischer Behandlung wie auch der zuverlässige Schutz vor z.T. gewalttätigen Diskriminierungen weiterhin Desiderate dar.

\subsection{Großbritannien}

Ganz anders präsentiert sich die Situation transsexueller Menschen mittlerweile in Großbritannien: Der Umgang mit Transsexualität wird im Vereinigten Königreich durch den Gender Recognition Act vom 8.6.2004 geregelt, der am 1.4.2005 in Kraft trat. ${ }^{44}$ Dieses Gesetz gilt - zumindest aus der Sicht vieler Betroffener - europaweit als beispielhaft. ${ }^{45}$ Hiernach können transsexuelle Menschen nach der rechtlichen Anerkennung des Geschlechtswechsels im neuen Geschlecht die Ehe mit einer Person des anderen Geschlechts eingehen. Die Neuformulierung der gesetzlichen Rahmenbedingungen wurde katalysiert bzw. flankiert von sehr weit gehenden Forderungen britischer Selbsthilfevereinigungen.

Der Gender Recognition Act setzt auch Maßstäbe beim Schutz vor Diskriminierung transsexueller Personen: Bereits in der Phase der geschlechtsanpassenden Maßnahmen können Betroffene sich aufgrund der aktuellen Gesetzgebung auf den Schutz vor Diskriminierung aus Gründen des Geschlechts berufen: ${ }^{46}$ Dies betrifft vor allem den Problemkreis Mobbing und Diskriminierung am Arbeitsplatz. ${ }^{47}$ Wenngleich die Betroffenen de facto auch nach der Anerkennung des Wunschgeschlechts stigmatisiert werden können, verfügen sie über ein verbrieftes Klagerecht:

„Im Rahmen von Diskriminierungen aufgrund des Geschlechts am Arbeitsplatz ist nach Reformierung des Sex Discrimination Act von 1975 im Jahr 1999 inzwischen anerkannt, dass transsexuelle Personen, egal in welchem Stadium der Geschlechtsanpassung, Klagen aufgrund einer Diskriminierung aufgrund des Geschlechts geltend machen können, wenn sie in einer Geschlechtsanpassung begründet liegen. ${ }^{“ 48}$

Allerdings besteht trotz der liberalen Regelung in einzelnen Bereichen weiterhin Regelungsbedarf:

„Zu bemerken ist im weiteren ebenfalls, dass es sich bei den aufkommenden Problemen keinesfalls um Probleme handelt, die nur dem Vereinigten Königreich eigen sind, sondern

44 www.publications.parliament.uk/pa/ld200304/ldbills/004/2004004.pdf.

45 www.tgnews.de/news/index.php?shownews=211: „Mit dieser modernen Regelung setzt Großbritannien einen Meilenstein für ein modernes TransGender-Gesetz, das auch für die dringend anstehende Novellierung des vor 20 Jahren fortschrittlichen, heute aber völlig veralteten deutschen TransSexuellen-Gesetzes (TSG) Vorbild sein kann." [21.05.2007]

46 Cowan (2005), S. $77 \mathrm{f}$.

47 Darüber hinaus sind transidente Personen allerdings weiterhin von zahlreichen sportlichen Wettbewerben ausgeschlossen: www.pfc.org.uk/campaign/pfcsprt01.htm [21.05.2007]. Dazu wird auf die mögliche Manipulationsmöglichkeit in Bezug auf den Hormonspiegel verwiesen. Im Gegensatz dazu dürfen transidente Personen unter bestimmten Voraussetzungen bei Olympischen Spielen seit Athen (2004) an den Start gehen, wohingegen Intersexualität zum Ausschluss führt: vgl. dazu Tolmein (2004).

$48 \operatorname{Moog}(2005)$, S. 51. 
dass es sich um Probleme handelt, die für alle Rechtsordnungen typisch sind, die gleichgeschlechtliche Ehen nicht anerkennen. “49

Der medizinische Umgang mit „Gender Dysphoria“ in Großbritannien ist von einer weitgehend auf die ratsuchenden Patienten abgestimmten Untersuchung und Behandlung geprägt. Die Therapie von Betroffenen, die sich häufig als „Transgenders“ bezeichnen, wird grundsätzlich im Rahmen des National Health Service geleistet. Allerdings ergab eine Umfrage aus dem Jahr 2002, dass nur ein geringer Prozentsatz der Betroffenen alle denkbaren Leistungen, begonnen bei der psychiatrischen Untersuchung bis hin zu einer (stimmangleichenden) phoniatrischen bzw. phonochirurgischen Therapie, erhält. ${ }^{50}$ Insgesamt sehen die britischen Transgender ihre Ansprüche jedoch weitgehend verwirklicht:

„Trans people will no longer be non-people, and the bill which parliament has passed is a very good one: it's possibly the best such legislation in the world. It offers privacy, it offers security of legal status, and - unlike most other countries - it does not exclude those people who have not been able to have genital surgery. ${ }^{~}{ }^{51}$

\title{
3.3 Frankreich
}

In Frankreich existiert kein Gesetz, das es einer Person in der Rolle und dem Erscheinungsbild des entgegengesetzten Geschlechtes erlaubt, seinen Familienstand zu ändern. Stattdessen wird die Entscheidung über die rechtliche Anerkennung des Geschlechtswechsels bewusst durch Gerichte vorgenommen. Die Begründung hierfür lautet:

\begin{abstract}
„Man hat angenommen, ein Gesetz müsse - einerseits - alle Rechtsprobleme der Transsexualität umfassend regeln, war und ist sich aber über die Regelung vieler dieser Probleme noch keineswegs klar; andererseits würde ein Gesetz neuen medizinischen Entwicklungen nur schwerfällig folgen können, die Gerichte könnten dies viel flexibler. ${ }^{\text {}}{ }^{2}$
\end{abstract}

Seit den 1990er Jahren fallen die Urteile zunehmend liberaler aus. Derzeit stellt sich die Situation für Betroffene folgendermaßen dar: Wenn ein mit derTherapie erfahrener Gutachter feststellt, dass eine transsexuelle Person nach einer medizinisch-chirurgischen Behandlung nicht mehr die Eigenschaften seines Ursprungsgeschlechts aufweist und zudem eine körperliche Erscheinung besitzt, die der des anderen Geschlechts näher steht, wird in der Regel eine Personenstandsänderung zugelassen. ${ }^{53}$ Gleichwohl müssen transsexuelle Bürger in Frankreich ein langwieriges und kostspieliges juristisches Verfahren durchlaufen. Der Rechtsweg transsexueller Personen beginnt mit dem Antrag auf Änderung ihres Vornamens. Dazu ist es notwendig, durch ein Gericht einen

49 Ebd.

50 Murjan/Shepherd/Ferguson (2002).

51 www.pfc.org.uk/pfclists/news-arc/2004q2/msg00087.htm [21.05.2007].

52 Puttfarken/Schnier (2004), S. 36.

53 Puttfarken/Schnier (2004), S. 37. 
Gebrauchsnamen auf den Papieren und einen neuen Vornamen eintragen zu lassen. Dies wird auf den Personendokumenten als „Rechtsübergangsphase“ vermerkt. Gegenwärtig werden die Anträge auf „Vornamensänderung“ in Frankreich beim Vormundschaftsgericht eingereicht. Die Dauer des Verfahrens beträgt drei bis sechs Monate. Kann der oder die Betroffene eine rechtliche Anerkennung der Geschlechtsänderung vorweisen, erfolgt die Vornamensanpassung allerdings automatisch..$^{54}$

Seit mehr als zwanzig Jahren obliegt die Diagnose „Transsexualität“ der psychiatrischen Fachdisziplin. Das Ziel der Psychiater besteht vor allem darin sicherzustellen, dass sich der Zustand des Patienten durch die Behandlung verbessert. Bei Zweifeln an einer Verbesserung soll eine Behandlung unterbleiben. Eine wesentliche Rolle spielen Informationsgespräche über die sexuelle Einstellung der Kandidaten. Dies führt beispielsweise unter anderem dazu, dass „Transfrauen“, die sich - mit Blick auf das „Zielgeschlecht“ Frau - als lesbisch empfinden, nicht zur Behandlung zugelassen werden. Andererseits wird vielen Betroffenen, die mit Blick auf das Ausgangsgeschlecht homosexuell orientiert sind, unterstellt, dass sie ihre sexuelle Veranlagung verleugnen und sich in die Rolle des/der „Transsexuellen“ flüchten. Darüber hinaus sind Personen, die mit Prostitution in Verbindung gebracht werden, grundsätzlich von der Behandlung ausgeschlossen. Dies wird von den Betroffenen als Diskriminierung kritisiert. ${ }^{55}$

Wenn die Diagnose „Transsexualität“ vorliegt, kann ein Betroffener eine so genannte „Demande d'Affection Longue Durée“ (A.L.D. ${ }^{56}$ bei der Sozialversicherung stellen. Die Diagnose einer Krankheit, die auf der Liste der A.L.D. enthalten ist, garantiert eine automatische Übernahme der Behandlungskosten. Im Fall von Transsexualität wird die Anerkennung des Antrags von Fall zu Fall entschieden, da die Diagnose nicht auf dieser Liste verzeichnet ist. Wenn die Diagnose gesichert ist, wird mit der Behandlung begonnen, die sich an den Internationalen Standards der HBIGDA orientiert. ${ }^{57}$

Wenngleich sich zahlreiche Chirurgen und andere Fachärzte in Frankreich für transsexuelle Menschen zuständig erklären, gibt es nur wenige erfahrene multidisziplinäre Behandlungsteams.${ }^{58}$ Vor diesem Hintergrund kann es nicht überraschen, dass viele Betroffene ins Ausland ausweichen, um sich operieren zu lassen.

Zwischenzeitlich haben Betroffenenverbände ein Communiqué erarbeitet, das auf diverse Missstände in der Behandlung hinweist, aber auch auf die Optimierung der Zusammenarbeit abzielt. Einige der vorgetragenen Forderungen der konzertierten Aktion der Verbände sind ein Zugang zu chirurgischer Behandlung ohne stigmatisierende psychiatrische Diagnose analog zum Britischen Gender Recognition Act sowie das Recht auf einen geschlechtsange-

54 Puttfarken/Schnier (2004), S. $37 \mathrm{f}$.

55 Ebd.

56 „Zuwendungsantrag auf lange Frist“ (übersetzt v. d. Verf.).

57 www.wpath.org/publications_standards.cfm [21.05.2007].

58 Die medizinische Zulassung von Heilverfahren und ihre Standardisierung liegt in Frankreich in den Händen der ANAES (l'agence nationale d'accréditation et d'évaluation en santé): www.hassante.fr/portail/display.jsp?id=j_5 [21.05.2007]. 
passten Vornamen bzw. eine Geschlechtsanpassung in den Personalpapieren ohne vorausgehende chirurgische Maßnahmen. Allerdings treffen sie auch auf eine vom dichotomen Geschlechtermodell geprägte Gesellschaft, die derartigen Forderungen skeptisch gegenüber steht.

\section{Schlussbetrachtung}

Die untersuchten Staaten gehören ausnahmslos dem Europarat sowie der Europäischen Union an und unterliegen damit der Rechtssprechung durch den EGMR sowie der des EuCH. Gleichwohl ist der Umgang mit dem Phänomen Transsexualität innerhalb der Europäischen Union ausgesprochen disparat sowohl im Hinblick auf die rechtlichen Grundlagen als auch hinsichtlich der medizinischen Standards und der gesellschaftlichen Reaktionen.

Die neue Gesetzgebung in Belgien ist als Reaktion auf die (liberale) europäische Rechtssprechung zu verstehen. Die lange Verfahrensdauer von einem ersten Gesetzentwurf im Jahr 2004 bis zur Verabschiedung 2007 gibt allerdings einen Hinweis auf den erheblichen Diskussions- und Klärungsbedarf, den die gesetzliche Neuregelung mit sich brachte. Es ist davon auszugehen, dass das „Loi relative à la Transsexualité" zu einem liberaleren Umgang mit Transsexualität führen wird. Zugleich ist einer stärkeren Standardisierung und Operationalisierung des in juristischer Hinsicht bisher nur grob formalisierten Verfahrens Grund gelegt. Inwieweit das Gesetz allerdings in praxi aus Betroffenensicht Erleichterungen bringt, muss die nähere Zukunft zeigen.

In Belgien gilt im Übrigen, was der EGMR für alle Mitgliedsstaaten der Europäischen Union verbindlich festgestellt hat: Die freie Verfügbarkeit über die eigene Person lässt juristisch nur eine Möglichkeit offen: Das Phänomen Transsexualität ist im Grundsatz anzuerkennen.

\section{Literatur}

Association For The Study And Defence Of The Right To The Genre Identity: The Portuguese Transgender Community: An Unknown Reality, http://a-trans. planetaclix.pt/english_documentation/documentation.htm [21.05.2007]

Association For The Study And Defence Of The Right To The Genre Identity: The image of transsexuality and is connection to the HIV virus, http://tgeu.net/Documents/p_ATrans_Bangkok.pdf

Associação ILGA Portugal (o. J.): Associação ILGA Portugal, Situação Geral Das Pessoas Transexuais Em Portugal, www.ilga-portugal.pt/glbt/gip/transexualidade. htm\#SITUA [21.05.2007]

Becker et al. (1997): Sophinette Becker, Hartmut A. G. Bosinski, Ulrich Clement et al., Standards der Behandlung und Begutachtung von Transsexuellen der Deutschen Gesellschaft für Sexualforschung, der Akademie für Sexualmedizin und der Gesellschaft für Sexualwissenschaft, Zeitschrift für Sexualforschung 10 (1997), 2, S. 147-156

Becker et al. (1998): Sophinette Becker et al., Es gibt kein richtiges Leben im Falschen. Antwort auf die Kritik an den ,Standards der Behandlung und Begutachtung von Transsexuellen', Zeitschrift für Sexualforschung 11 (1998), S. 155-162

Bréton et al. (1985): Jacques Bréton et al., Le transsexualisme: étude nosographique et médico-légale, Rapport de médecine légale, congrès de psychiatrie et de neurologie, Besançon 1985, Paris 1985

Castel (2001), Pierre-Henri Castel, Algumas reflexões para estabelecer a cronologia do „fenômeno transexual“ (1910-1995), Revista Brasileira de História 21 (2001), 41, p. 77-111 
Chiland (1997): Collette Chiland, Changer de sexe, Paris 1997

Coelho de Lima (2001): João Coelho de Lima, Transexualidade, Identidade e Casamento - Alguns Problemas, Scientia luridica 50 (2001), p. 125-156

Cohen-Kettenis/Pfäfflin (2003): Peggy Cohen-Kettenis, Friedemann Pfäfflin, Transgenderism and Intersexuality in Childhood and Adolenscence. Making Choices, Thousand Oaks, London, New Delhi 2003

Costa-Santos/Madeira (1996): Jorge Costa-Santos, Rosa Madeira, Transsexualism in Portugal: The legal framework and procedure, and its consequences for transsexuals, Medicine, science and law 36 (1996), 3, p. 221-225

Cowan (2005): Sharon Cowan, Gender is no Substitute for Sex: a Comparative Human Rights Analyses of the Legal Regulation of Sexual Identity, Feminist Legal Studies 13 (2005), p. 67-96

Da Silva (2007): Adrian Da Silva, Zur Konstruktion von Geschlecht und Geschlechterregimen in dem Gender Recognition Act und im englischen Parlament, liminalis 1, 1 (2007), S. 83-108

De Cuypere/Jannes/Rubens (1995): Griet de Cuypere, C. Jannes, Robert Rubens, Psychosocial functioning of transsexuals in Belgium, Acta Psychiatrica Scandinavica 91 (1995), p. 180-184

Deutsche Gesellschaft für Sexualforschung, Akademie für Sexualmedizin und Gesellschaft für Sexualwissenschaft (1998): Deutsche Standards der Behandlung und Begutachtung von Transsexuellen, The International Journal of Transgenderism, 2 (1998), 4, www.symposion.com/ijt/ijtc0603d01.htm

Dörner (1988): Günter Dörner, Neuroendocrine response to estrogen and brain differentiation in heterosexuals, homosexuals and transsexuals, Archives of Sexual Behaviour 17 (1988), p. 57-75

Eckl (2004): Christian Eckl, Portugal, in: Jürgen Basedow, Jens M. Scherpe (Hrsg.), Transsexualität, Staatsangehörigkeit und internationales Privatrecht (= Studien zum ausländischen und internationalen Privatrecht, 134), Tübingen 2004, S. $59 \mathrm{f}$.

Ellger (2004): Reinhard Ellger, Vereinigtes Königreich, in: Jürgen Basedow, Jens M. Scherpe (Hrsg.), Transsexualität, Staatsangehörigkeit und internationales Privatrecht (= Studien zum ausländischen und internationalen Privatrecht, 134) Tübingen 2004, S. 80-91

Faculdade de Medicina de Lisboa (2004): Sexualidade Humana, Revista da Faculdade de Medicina de Lisboa, Série III, Vol. 9, Supl. 1, Maio 2004

Greif (2005): Elisabeth Greif, Doing Trans/Gender. Rechtliche Dimensionen (= Linzer Schriften zur Frauenforschung, 29), Linz 2005

Groß/Steinmetzer (2007): Dominik Groß, Jan Steinmetzer: Transsexualität zwischen Medizin, Recht und Ethik. Ein europäischer Vergleich, in: B. Sharon Byrd, Jan C. Joerden (Hrsg.), Jahrbuch für Recht und Ethik, Band 14, 2006/07, S. 581-609

HBIGDA (2001), Harry Benjamin International Gender Dysphoria Association (ed.), Standards of Care for Gender Identity Disorders (SoC), 6. Version von 2001, www.wpath.org/Documents2/socv6.pdf

Kelly (2001): Donna Patricia Kelly, Estimation of the Prevalence of Transsexualism in the UK, October 13, 2001, http://ai.eecs.umich.edu/people/conway/TS/UK-TSprevalence.html [21.05.2007]

Madeira (1999): Rosa Madeira, Mudança de Identidade/Transexualismo, Dissertação de Mestrado em Medicina Legal da Faculdade de Medicina da Universidade de Coimbra 1999

Matos (2004): Manuel Godinho de Matos: A Cirurgia da Inter e da Transsexualidade, Faculdade de Medicina de Lisboa (2004), p. 91-98

Meningaud (1998): Jean-Paul Meningaud, Problèmes éthiques posés par la demande chirurgicale de redétermination de sexe chez le transsexuel, présenté 7.09.1998, http://infodoc.inserm.fr/ethique/Travaux.nsf/ ob18553bd754a1c7c1256701002c6600/41617823c0a98147c12567c800321254?0penDocument

Moog (2005): Bettina Charlotte Moog: Das ,Gespenst' der Gleichgeschlechtlichkeit - Transsexuelle zwischen Anerkennung und Diskriminierung, Masterthesis, Wien 2005

Murjan/Shepherd/Ferguson (2002): Sarah Murjan, Michelle Shepherd, Brian G. Ferguson, What services are available for the treatment of transsexuals in Great Britain?, Psychiatric Bulletin 26 (2002), p. 210-212

Pechorro/Vieira (2004): Pedro Pechorro, Rui M. X. Vieira, Avaliação Psicológica de um Grupo de Transexuais com Indicação para Cirurgia de Reatribuição de Sexo. Estudo preliminar, Faculdade de Medicina de Lisboa (2004), p. 77-84

Pfäfflin, Friedemann, Erstellung der Gutachten, in: Ulrich Clement, Wolfgang Senf (Hrsg.): Transsexualität, Stuttgart, New York 1996, S. 80-87 
Pfäfflin (2006): Friedemann Pfäfflin, Soziale Probleme, in: Günter Stalla, Therapieleitfaden Transsexualität, Bremen 2006, S. 64-68

Pintens (2004): Walter Pintens, Belgien, in: Jürgen Basedow, Jens M. Scherpe (Hrsg.), Transsexualität, Staatsangehörigkeit und internationales Privatrecht (= Studien zum ausländischen und internationalen Privatrecht, 134), Tübingen 2004, S. 20-26

Pintens/Scherpe (2003): Walter Pintens, Jens M. Scherpe, Gleichgeschlechtliche Ehen in Belgien, Das Standesamt 56 (2003), S. 321-324

Pintens/Scherpe (2004): Walter Pintens, Jens M. Scherpe, Gleichgeschlechtliche Ehen im belgischen internationalen Privatrecht, Das Standesamt 57 (2004), S. 290-292

Puttfarken/Schnier (2004): Hans-Jürgen Puttfarken, Judith Schnier, Frankreich, in: Jürgen Basedow, Jens M. Scherpe (Hrsg.), Transsexualität, Staatsangehörigkeit und internationales Privatrecht (= Studien zum ausländischen und internationalen Privatrecht, 134), Tübingen 2004, S. 36-40

Ramm (2003): Wiebke Ramm, Die „Disziplinierung“ des „transsexuellen Subjekts“. (Re)Produktion normierter Zweigeschlechtlichkeit im institutionalisierten Geschlechtswechsel, Forum Kritische Psychologie 46 (2003), S. 82-100

Reucher (2002): Tom Reucher, Ethnopsychiatrie, théorie queer et „transsexualisme“ (syndrome de benjamin): pratiques cliniques, mémoire de DESS“, Paris 2002, http://syndromedebenjamin.free.fr/textes/ travauxfac/memoiredesstom/chap4.htm

Sandland (2005): Ralph Sandland, Feminism and the Gender Recognition Act 2004, Feminist Legal Studies 13 (2005), p. 43-66

Steinmetzer/Groß/Duncker (2007): Jan Steinmetzer, Dominik Groß, Tobias Heinrich Duncker, Ethische Fragen im Umgang mit transidenten Personen - Limitierende Faktoren des gegenwärtigen Konzepts von „Transsexualität", Ethik in der Medizin 19 (2007), 1, S. 39-54

Tolmein (2004): Oliver Tolmein, Sie werden auch Wagen lenken. Transsexuelle bei Olympia, Frankfurter Allgemeine Zeitung 199 (27.08.2004), S. 44

TransStreetDay (2005): TransStreetDay, Die rechtliche Situation transsexueller und transidenter Menschen in Europa, Vortrag TransStreetDay, Dresden 2005, www.4ftm.de/ppt/rechtliche_situation_ts_europa.ppt [11.04.2006; nicht mehr verfügbar]

Vieira (2004): Rui M. X. Vieira, Transexualismo: da Clínica ao Diagnóstico, Faculdade de Medicina de Lisboa (2004), p. 85-90

Wachsmann (2003): Patrick Wachsmann, La folie dans la loi: considérations critiques sur la nouvelle jurisprudence de la Cour européenne des droits de l'homme en matière de transsexualisme en marge des arrêts Christine Goodwin c. le Royaume-Uni et I. c. le Royaume-Uni du 11 juillet 2002, Revue trimestrielle des droits de l'homme 14 (2003), 56, p. 1157-1183

Whittle (2006): Stephen Whittle, Jumping the hurdles of Gender - Transsexuals finally make legal headway, www.pfc.org.uk/node/364 [23.05.2007]

\section{Urteile und Gesetzestexte:}

\section{Europa}

ABI 1989 C 256/33 (Europäisches Parlament)

EGMR, Serie A n² 231 C, „B. gegen Frankreich“, Rs. 57/1990/248/319

EuGH Rs C-13/94 „P. gegen S. und Cornwall County Council“

\section{Großbritannien}

Rechtssache C-117/01 K. B. gegen The National Health Service Pensions Agency und The Secretary of State for Health zur "Gleichbehandlung von Männern und Frauen - Ausschluss eines Transsexuellen von einer dem überlebenden Ehegatten vorbehaltenen Witwerrente-Grundrecht auf Eheschließung", http://europa.eu.int/ jurisp/cgi-bin/gettext.pl?lang=de\&num=79969389C19010117\&doc= T\&ouvert= T\&seance=CONCL\&where=() [21.05.07] 
Gender Recognition Bill: www.publications.parliament.uk/pa/ld200304/ldbills/004/2004004.pdf Corbett vs. Corbett, in: The Law Reports. Probate, Divorce, and Admiralty Division 1971, p. $83 \mathrm{ff}$.

Matrimonial Cause Act von 1973, www.terry.co.uk/matcaus.html

Judgments - Bellinger (FC) (Appellant) v. Bellinger, www.publications. parliament.uk/pa/ld200203/ldjudgmt/ jd030410/bellin-1.htm

Sarah Margaret Richards vs. Secretary of State for Work and Pensions, http://europa.eu.int/eur-lex/lex/ LexUriServ/LexUriServ.do?uri =0|:C:2006:143:0013:0013:EN:PDF

Civil Partnership Act 2004, www.opsi.gov.uk/ACTS/acts2004/20040033.htm

\section{Portugal}

Colectânea de Jurisprudência 1986 IV, p. 123 f.

Colectânea de Jurisprudência 1984 II, p. $124 \mathrm{f}$.

Colectânea de Jurisprudência 1993 V, p. 118

Colectânea de Jurisprudência 1997 II, p. 27 f.

\section{Frankreich}

Vollversammlung des Kassationsgerichtshofs vom 11.12.1992, Procédure: René X. Cassation sans renvoi, nach vorausgegangener Entscheidung des Gerichts Aix-en-Provence vom 15.11.1990, http://perso.orange.fr/ gd.melison/jp/famille/Cassplen_19921211.htm; auch verfügbar unter: Bulletin civil $n^{\circ} 13$; Gazette du palais 1993, p. 180

\section{Deutschland}

BVerfG, 1 BvL 1/04 vom 18.7.2006

Gesetz über die Änderung der Vornamen und die Feststellung der Geschlechtszugehörigkeit in besonderen Fällen (Transsexuellengesetz - TSG), Bundesgesetzblatt, Jahrgang 1980, Teil I, Fortlaufende Seiten 1654-1658 vom 10.09 .1980 